\title{
Failure of nifedipine treatment in primary pulmonary hypertension
}

Sir,

In an attempt to emulate the success described by Camerini $e t$ al. in a recent article in the British Heart Journal, ${ }^{1}$ we tried nifedipine in the treatment of a 36-year-old woman who had both clinical and haemodynamic findings of primary pulmonary hypertension.

The patient was studied using a Swan-Ganz catheter; the procedure used by Camerini et al. was not changed. The following results were obtained:

Only diazoxide at the lowest dose appeared to decrease total pulmonary resistance significantly. Nifedipine failed to do so. Other trials with molsidomine (a new long acting vasodilator) and hydralazine were also unfruitful.

As proposed by Klinke, ${ }^{2}$ drugs used to attempt to treat primary pulmonary hypertension need acute testing before being started on a long term basis; moreover, monitoring of the pulmonary vascular pressure is mandatory to avoid aggravating the condition. We would like to emphasise that whereas primary pulmonary hypertension may occasionally be ameliorated by various drugs, this response is individual and not the rule.

\author{
G Berkenboom, J Sobolski, E Stoupel, \\ Department of Cardiology (Degre S.), \\ Erasme Hospital, \\ Free University of Brussels, \\ Brussels, Belgium.
}

Note: This is a further letter to correspondence published in $\mathrm{Br}$ Heart $\mathcal{F}$ 1981; 46: 230-1. Dr Camerini was shown this letter but thought that his earlier letter covered the points now raised in this one by Berkenboom et al.

\section{References}

1 Camerini F, Alberti E, Klugmann S, Salvi A. Primary pulmonary hypertension: effects of nifedipine. Br Heart f 1980; 44: 352-6.

2 Klinke WP. Treatment for primary pulmonary hypertension. Am Heart $\mathcal{F}$ 1980; 100: 587-8.

\begin{tabular}{|c|c|c|c|c|c|c|}
\hline \multicolumn{2}{|c|}{$\begin{array}{l}\text { 1. Nifedipine (20 } \mathrm{mg} \text { sublingually) } \\
\text { HR } \\
\text { (beats/min) }\end{array}$} & $\underset{(I / m i n)}{C O}$ & $\begin{array}{l}\bar{P} A \\
(m m H g)\end{array}$ & $\begin{array}{l}\text { TPR } \\
\left.\text { (dyne s } \mathrm{cm}^{-5}\right)\end{array}$ & $\begin{array}{l}\bar{P} a \\
(m m H g)\end{array}$ & $\begin{array}{l}\text { TSR } \\
\left.\text { (dyne s } \mathrm{cm}^{-5}\right)\end{array}$ \\
\hline $\begin{array}{l}\text { Basal } \\
30^{\prime} \\
60^{\prime}\end{array}$ & $\begin{array}{r}98 \\
102 \\
104\end{array}$ & $\begin{array}{l}4.3 \\
4.8 \\
4.8\end{array}$ & $\begin{array}{l}50 \\
59 \\
59\end{array}$ & $\begin{array}{l}929 \\
982 \\
982\end{array}$ & $\begin{array}{l}92 \\
91 \\
87\end{array}$ & $\begin{array}{l}1709 \\
1697 \\
1614\end{array}$ \\
\hline 2. Diazo & $\begin{array}{l}\text { ulmonary artery) } \\
\text { HR } \\
\text { (beats/min) }\end{array}$ & $\begin{array}{l}C O \\
(1 / \text { min })\end{array}$ & $\begin{array}{l}\bar{P} A \\
(m m H g)\end{array}$ & $\begin{array}{l}\text { TPR } \\
\left(\text { dyne s } \mathrm{cm}^{-s}\right)\end{array}$ & $\begin{array}{l}\bar{P} a \\
(m m H g)\end{array}$ & $\begin{array}{l}\text { TSR } \\
\text { (dyne } \mathrm{cm}^{-5} \text { ) }\end{array}$ \\
\hline $\begin{array}{l}\text { Basal } \\
50 \mathrm{mg} \\
100 \mathrm{mg} \\
100 \mathrm{mg}\end{array}$ & $\begin{array}{l}115 \\
125 \\
132 \\
137\end{array}$ & $\begin{array}{l}3.5 \\
4.05 \\
4.35 \\
4.35\end{array}$ & $\begin{array}{l}62 \\
55 \\
62 \\
61\end{array}$ & $\begin{array}{l}1415 \\
1085 \\
1138 \\
1120\end{array}$ & $\begin{array}{l}85 \\
90 \\
87 \\
82\end{array}$ & $\begin{array}{l}1940 \\
1775 \\
1598 \\
1506\end{array}$ \\
\hline
\end{tabular}

HR, heart rate; $\mathrm{CO}$, cardiac output; $\overline{\mathrm{P}} \mathrm{A}$, mean pulmonary arterial pressure; $\overline{\mathrm{P}}$, mean systemic arterial pressure; TSR, total systemic resistance; TPR, total pulmonary resistance. 